Response to Commentaries on Precis of Cognitive Gadgets

Behavioral and Brain Sciences

AAM, 20 March 2019

\title{
COGNITION BLINDNESS AND COGNITIVE GADGETS
}

\author{
Cecilia Heyes \\ All Souls College \& Department of Experimental Psychology \\ University of Oxford \\ Oxford OX1 4AL \\ United Kingdom
}

cecilia.heyes@all-souls.ox.ac.uk

\begin{abstract}
Responding to commentaries from psychologists, neuroscientists, philosophers and anthropologists, I clarify a central purpose of Cognitive Gadgets - to overcome 'cognition blindness' in research on human evolution. I defend this purpose against Brunerian, extended mind, and niche construction critiques of computationalism, that is, views prioritising meaning over information, or asserting that behaviour and objects can be intrinsic parts of a thinking process. I argue that empirical evidence from cognitive science is needed to locate distinctively human cognitive mechanisms on the continuum between gadgets and instincts. Focussing on that requirement, I also address specific challenges, and applaud extensions and refinements, of the evidence surveyed in my book.
\end{abstract}


It has been said that "a writer's idea of sound criticism is ten thousand words of closely reasoned adulation". I cannot disagree with this untraceable wag, but the 30 commentators on Cognitive Gadgets (CG) provided some 30,000 words of criticism that are of much greater scientific value than adulation. I am grateful to them all. The response that follows is V-shaped. It starts with the broadest conceptual and methodological issues and funnels down to matters arising from specific empirical studies.

\section{R1 Cognition blindness}

One of the overarching aims of $C G$ is to encourage people interested in human evolution to think not only about brains, bodies, behaviour and beliefs, but in a computational way about how our minds work. I was trying to overcome 'cognition blindness', a tendency among evolutionists to look straight past an important resource - the kind of cognitive science, thriving in labs all over the world since the 1970s, that casts mental processes as software running on the brain (Block 1995). Some commentators revealed inadvertently just how tenacious cognition blindness can be. While making otherwise valuable points, these commentators looked straight past the software and wrote about cognitive gadgets as if they are parts of the brain, chunks of behaviour, or airy bridges between brain and behaviour built out of folk psychology and pure maths (e.g. Badcock et al, lannetti \& Vallortigara, Jablonka et al, Smaldino \& Spivey, Sperber, Tennie, Whiten). The arcuate fasciculus is part of the brain not of the mind. 'Social organisation', 'norm', 'conformity' and (in frequent usage) 'decision rule' refer to behavioural regularities rather than computational processes. And in many models, terms such as 'inference' and 'belief' are taken from folk psychological stock and applied so promiscuously that they lose all meaning, leaving maths to do the work.

Of course it is vital to study the brain and behaviour, often with the help of mathematical models and folkweave characterisations of the mind, but $C G$ recommends a major addition to the evolutionist's armoury. It suggests that we can better understand human evolution if we recognise that the brain interacts with behaviour via cognitive mechanisms; these mechanisms are among the 
targets of genetic and cultural selection; and folk psychology seldom provides the most precise and empirically-grounded descriptions of how these mechanisms work. In many cases - such as the mechanisms involved in object recognition, speech production, and reading - folk psychology is simply silent. Mathematical models can help to fill the silence, but without more abstract, software characterisations of what the mind is doing these models struggle to make testable predictions (Coltheart 2002; 2012).

Dominey and Fenici \& Garofoli certainly do not suffer from cognition blindness. They see the computationalism of $C G$ clearly and challenge it head-on. At the heart of their challenge are a lament and an historical claim. They lament that computationalist cognitive science makes little contact with lived experience and therefore with the humanities. It sides with the natural sciences, offering 'explanation' rather than 'understanding', in the language of 'information' rather than of 'meaning'. The historical claim is that this could easily have been otherwise. If computer technology had not been advancing so rapidly when behaviourism ran out of steam, the cognitive revolution would have produced a more humane, meaning-based cognitive science (Bruner 1990)

I am sympathetic to the lament and I find the historical claim fully plausible. It is deeply regrettable that we are still a long way from knowing how to integrate explanation and understanding, information and meaning, science and the humanities. However, I doubt that the direction of cognitive science can be changed from on-high by the kind of metaphysical arguments advanced by critics of computationalism (e.g. Baggs et al; Clark \& Chalmers, 1998; Hutto \& Myin 2017, Malafouris 2016), and, even if such inorganic change was possible, I am not sure there would be a net gain from switching sides. A cognitive science that jettisoned computationalism for 'meaning' would lose most of the insights accumulated over the last 50 years and, although closer to the humanties, would be alienated from the natural sciences. As long as a meaning-based approach continues to dominate social and developmental psychology (e.g. Tomasello 2009; 2014; Whiten), I see no danger that it will be abandoned completely by those who study the mind. Furthermore and this may be where I differ most from Dominey, and Fenici \& Garofoli - I am not disturbed by the 
historically contingent origins of computationalism. I see both computationalism and folk or 'beliefdesire' psychology - the 'meaning' framework - as products of cultural evolution. They each have strengths and weaknesses, and are eminently revisable. For now, as highlighted by van Bergen \& Sutton, there are advantages to be gained from using both folk psychology and computationalism to understand the evolution of the human mind.

\section{R2 Grist and mills}

In one of my efforts to overcome cognition blindness, to point at what is missing from cultural evolutionary studies, I borrowed an 800-year-old metaphor of the mind from Saint Thomas Aquinas. I said that cultural evolution operates not only on the grist of the mind (e.g. beliefs, ideas, behaviours, skills, artefacts) but also on the mills (cognitive mechanisms). Like most metaphors, this one is far from perfect. Mills work on grist and cognitive mechanisms work on beliefs, ideas, behaviours, skills and artefacts (BIBSA); cognitive mechanisms take these particulars as input and transform them. So far, so good. But whereas mills turn grist into flour, cognitive mechanisms turn BIBSA into more BIBSA. Beliefs, ideas, behaviours, skill and artefacts - the usual targets of cultural evolutionary analysis - are both inputs and outputs of cognitive processing.

As Smaldino \& Spivey noticed, the grist and mills metaphor would have been even more imperfect if I had used it to capture, not the synchronic relationship between cognitive processes and their contents, but the diachronic relationship between social interactions and neural mechanisms. I agree with them that "the social mechanisms of language use and the neural mechanisms of language processing may not be well treated as 'a grist' and 'a mill', respectively". Fortunately, although the mutually formative relationship between social interactions and cognitive (rather than neural) mechanisms was a central theme of CG, I did not try to capture that relationship with a metaphor of any kind. Instead I characterised it as a relationship in which cognitive mechanisms undergo cultural evolution. 
I am pleased to find that I have much in common with Baggs, Raja and Anderson, but they are also unhappy about the grist and mills metaphor. At first blush it seems that, in their view, this metaphor misled me into thinking that "the things we do and make" are mere products of cognitive processes. On this reading, to cast behaviour and artefacts as grist is to overlook the vital role of the agent's own behaviour in determining the information to which $\mathrm{s} /$ he has access, and to underestimate the importance of both artefacts and the behaviour of other agents as carriers of information in their own right. But when the first blush has subsided, this is an implausible reading of the concern expressed by Baggs et al. CG does not say a lot about artefacts because it focusses on social cognition (language, mindreading, imitation) rather than instrumental cognition (e.g. causal understanding, spatial navigation), but it dwells at great length on the importance of social interaction - what we do with others - in informing and shaping the human mind. Given this emphasis, it is more likely that Baggs et al are objecting to the metaphysics of the grist and mills metaphor. They are challenging the assumption - enshrined in both computationalism and contemporary Western folk psychology - that thinking, acting and artefacts are three fundamentally different kinds of things. They see value in the idea of "the extended mind" (Clark \& Chalmers 1998), the view that behaviour and objects can be intrinsic parts of a thinking process.

There is something exhilarating about philosophical work on the extended mind. Consistent with the cultural evolution of mindreading, it shows that our thinking about thinking could easily have been both coherent and radically different from the way it is now. However, call me oldfashioned, I cannot see what would be gained, in everyday life or in cognitive science, by switching from the view that the mind is 'in the head' to the view that the mind is (partly) in the world. The capacity of a puddle to constrain dance movements and inspire mischief can be captured not only by casting the puddle as "a component in our action control" (Baggs et al), but also in the conventional way by casting the puddle as an environmental input to action control - grist to a mill. Similarly, in the diachronic case, when I say that the childhood development of imitation draws on experience with optical mirrors, and of being imitated by others, I struggle to see what would be gained by 
casting the mirrors and the actions of other agents as component parts of the child's developing mind. It is kind of cool to think of it that way, but would the extended mind perspective suggest different empirical questions, or make existing questions more empirically tractable?

Baggs et al also chide me (gently) for neglecting niche construction, "the idea that animals reshape their environments through their actions, and this in turn structures the selection pressures exerted on current and future generations". It is not clear whether niche construction is a bold new concept, like the extended mind, or a catchy new term for an important and pervasive phenomenon that has long been recognised by evolutionists (Gupta et al. 2017; Feldman, Odling-Smee \& Laland 2017). Without attempting to resolve that issue, which is way above my pay-grade, I can only say that I am puzzled when people suggest that niche construction - a ubiquitous phenomenon throughout the animal kingdom - is not just important in humans, but a key to understanding distinctively human characteristics. It is a bit like the problem posed by research on social learning strategies in nonhuman animals (CG, Chapter 5). If most or all animals have social learning strategies / engage in niche construction, we need to find out what it is about human social learning strategies / niche construction that makes us different. Most of the explanatory work is done by the difference-maker rather than the base concept; in the case of social learning strategies, by the recognition that, in humans, some social learning strategies are explicitly metacognitive.

\section{R3 Gadgets and Instincts}

In the movies, Frankenstein screams maniacally "It's alive! It's alive!" as his monster begins to twitch. We have no trouble understanding what $\mathrm{Dr} F$. is asserting (and denying) even though he is drawing on a distinction, between life and death, that affords many intermediates and ambiguous cases. A creature can be more or less alive, closer or further away from death; there are entities viruses, zombies, Frankenstein's monster - that resist classification; and, like it says in The Book of Common Prayer, "In the midst of life we are in death". The distinction between cognitive gadgets and cognitive instincts, although less profound, is similarly sinuous. 
The first thing I should emphasise is that a cognitive gadget is not an entity "created by cultural evolution alone" (Whiten). As highlighted by Sperber, I am convinced that "The rich interactive complexity of developmental processes makes it absolutely clear that, in cognition as in other biological systems, there are no pure cases of nature or of nurture; no biological characteristic is caused only by 'the genes' or only by 'the environment'" (CG, p24). Rather, a cognitive gadget is a cognitive mechanism with distinctively human characteristics that have been shaped predominantly by selection operating on cultural variants. In contrast, a cognitive instinct is a cognitive mechanism with distinctively human characteristics that have been shaped predominantly by selection operating on genetic variants. The terms cognitive gadget and cognitive instinct mark the ends of a continuum of cases (Sperber), with, I argued in CG, imitation and mindreading close to the gadget end, and things like associative learning and the inborn face bias (lannetti \& Vallortigara) close to the instinct end of the continuum.

There are many evolutionary processes that could, in principle, send a cognitive process from one end of the continuum into a "messy middle ground" (Rathkopf $\&$ Dennett) between gadgetry and instinctiveness. For example, in principle, genetic assimilation (Del Giudice) could increase the role of genetically inherited information in shaping development, and genetic accommodation could amplify the roles of nature, nurture and/or culture (Jablonka et al). As Del Giudice underlined, this is not a "zero-sum competition". All of these in-principle possibilities I happily embrace. What puzzles me is that those commentators who were critical of the gadgetinstinct distinction seem to share my interest in examining how different factors (genetic, cultural, 'plasticity' etc) combine to produce cognitive development, but do not seem to believe that, in order to do this, one must be able to get an empirical handle on what and how each factor is contributing in any given case. It is as if they want to know how different ingredients and oven settings contribute to the texture and flavour of a cake, but do not believe that, to find out, one must be able to distinguish their contributions through intervention - e.g. by adding more flour - and by examining patterns of covariance - e.g. by comparing cakes baked at 180,190 and $200^{\circ} \mathrm{C}$. 
Badcock et al, Jablonka et al, and Sperber say very little about empirical matters. They distinguish types of interaction between genetic and experiential influences - or genetic and specifically cultural influences - without considering how the types could be distinguished in practice. For example, they do not explain how we would know whether genetic accommodation had or had not occurred (Jablonka et al), or how we can tell apart cases in which "A biological function [has been] fulfilled through the cultural evolution of an appropriate trait" and in which "cultural evolution [has taken] advantage of biologically evolved dispositions" (Sperber). On the other hand, Del Giudice, revisiting our disagreement about mirror neurons (Cook et al 2014; Del Giudice, Manera \& Keysers 2009), concerns himself with empirical matters but offers a counsel of despair. He doubts that twin studies can provide positive evidence of genetically inherited contributions to development, and remarks ominously that "It may be impossible to fully make sense of the cross-cultural data on developmental trajectories without addressing the thorny issue of national differences in cognitive ability". However, Del Giudice does not direct us to empirical methods that are, in his view, better able to trace the contributions of nature, nurture and culture to cognitive development. It seems that he wants to consigned cognitive mechanisms to the middle ground between gadgetry and instinctiveness because he despairs of us ever being able to find positive evidence of genetic, learning and cultural contributions.

As I acknowledge repeatedly in $C G$, both explicitly and by poring over data, it is very difficult indeed to get an empirical handle on the contributions of nature, nurture and culture to cognitive development. For example, after discussing a range of methods, I note:

"each of the methods outlined above is highly fallible. When learning opportunity A (e.g. to talk with a parent about mental states) correlates with cognitive ability $B$ (mindreading), it could be because a hidden factor, $C$ (linguistic skill), is influencing both $A$ and $B$, not because $A$ is causing $B$. Likewise, twin studies may indicate a relatively large genetic contribution to development simply because the people included in the study happen to have grown up in very similar environments, and, in crossspecies comparisons, convergent evolution can be mistaken for a strong influence of learning on development. Given these risks, in this area of science, as in most others, we have to place more trust in research that includes effective control procedures, and to look for convergent evidence - for signs that studies using different samples and methods are pointing to the same conclusion." (CG, p. 50) 
In my view, it is neither legitimate nor helpful to respond to these challenges with a 'messy middle default' i.e. by assuming that all three sources of information contribute about equally in all cases, or by assuming out of tribal loyalty (e.g. to behaviourism or High Church evolutionary psychology) that one of them is dominant. CG offers and uses a methodological template for parsing cognitive development, based on the distinction between poverty and wealth of the stimulus. I would be flabbergasted if this template was exactly right. It certainly needs refinement and to be augmented by modelling, especially non-linear modelling (Smaldino \& Spivey). But I shall stick to my guns on what the current evidence suggests - that many distinctively human cognitive mechanisms lie at the gadget end of the continuum (see below) - and, more generally, on the necessity for empirical evidence from cognitive science to back up claims about the roles of nature, nurture and culture in cognitive development. I will be content if CG proves to be "a timely provocation" (Del Giudice) in this respect; if it encourages those interested in human evolution to recognise that claims about the innateness and genetic assimilation of cognitive processes are not helpful unless they are backed by specific, discriminative empirical evidence. We should not allow nativism to be a matter of taste.

\section{R4 More about gadgets}

Before turning to the evidence surveyed in $C G$, I would like to say a little more about what I had in mind when I coined the term 'cognitive gadgets'. (Gadgets are out in the world now, so people can make of them what they will, but I still feel a bit proprietory.)

First, I have been convinced by Buskell (2018) that 'minority' cognitive processes - such as those specialised for chess (Del Giudice), lace making, or abacus calculation - are cognitive gadgets in good standing, and that they could prove to be a valuable resource in empirical research on the cultural evolution of human cognition. However, following High Church evolutionary psychology, I am especially interested in the types of cognitive mechanisms - such as mindreading, episodic memory, language, imitation - that are present in most people alive today. These human-naturedefining cognitive gadgets are, for me, the paradigmatic cases. Note, with Badcock et al, that many 
people who now identify as 'evolutionary psychologists' are not High Church. I may even be one of them. But, of course, insofar as the departure from orthodoxy involves rejection of computationalism, I regard it as heresy.

Second, Del Giudice and Sperber take me to be yet more devout about associative learning than I really am. I see associative learning as a powerful engine, but not the only engine, in the construction of cognitive gadgets. As I tried to make clear in my discussions of metacognitive social learning strategies and mindreading (CG, Chapters 5 and 7), like Dominey, I regard language as another major generator.

Finally, I want to put my hands up and acknowledge that, although it suggests that cognitive gadgets are shaped by cultural group selection, the book says relatively little about evolutionary dynamics (Del Guidice, Smaldino \& Spivey). It is the work of a cognitive scientist interested in evolution, not of an evolutionist interested in cognitive science. I hope researchers with complementary expertise will take up the challenge, using modelling and historical-anthropological data to assess the plausibility of the hypothesis that distinctively human cognitive mechanisms (along with grist - social organisation, norms, beliefs etc) have been shaped by cultural selection. In the meantime, let me reiterate baldly an argument in favour of cultural selection that did not make it from the book to the precis: We know of three sources of adaptive fit between a species-typical trait and its environment - intelligent design, genetic selection and cultural selection (Dennett 2017). Intelligent design now contributes to the development of some distinctively human cognitive mechanisms (e.g. there are education programmes designed to promote literacy), but it is not a plausible candidate for most of these mechanisms (e.g. mindreading, imitation). Genetic selection is the option backed by High Church evolutionary psychology, but, I argue in CG, contemporary evidence from cognitive science is not consistent with the idea that genetic selection is the principal architect of the human mind. Therefore, to the extent that distinctively human cognitive mechanisms are adaptive - do their jobs well - it must be because they have been shaped by the third designer, cultural selection. 


\section{R5 Evidence}

\section{R5.1 Starter kit}

\section{R5.1.1 Face preference}

lannetti \& Vallortigara draw attention to a very interesting, recently published electroencephalographic study showing a stronger neural response to upright than inverted face-like stimuli in newborns (Buiatti et al. 2019). At first I could not work out why lannetti \& Vallortigara regard this study as contrary to my suggestion that an inborn face bias is part of the genetically inherited starter kit for distinctively human cognition. The results are entirely consistent with the behavioural evidence on which I based this claim, showing that newborns have an attentional bias in favour of face-like stimuli. Having read lannetti and Vallortigara's commentary more carefully, I think there has been a misunderstanding due to their focus on the brain (hardware) and my focus on cognition (software). They identify the inborn face bias with a particular neural response. For them, the inborn face bias is a neural response. Therefore, by definition, as this response declines the inborn face preference goes away; it is a transitory phenomenon rather than something that persists to become part of mature face processing. In contrast, for me the inborn face bias is a functional entity observed at a particular stage in development; it is whatever makes newborns attend more to face-like stimuli. On this cognitive view, the decline of a particular neural response in the first few days post-partum is entirely consistent with the inborn face bias being a foundation for growth, via domain-general learning, of more specific face-related attentional biases.

\section{R5.1.2 Executive functions}

In their commentary based on careful reading of $C G$ and packed with interesting data, Braem \& Hommel challenge my suggestion that enhanced executive functions are part of the genetic starter kit for distinctively human cognition. Instead, they (and now I) find it plausible that, insofar as inhibitory control, working memory and cognitive flexibility are more advanced in humans than 
other animals, it is due to genetically-based changes in associative learning plus sociocultural input during development. I found myself wondering, if this is correct, how free-living nonhuman animals could get enough of the right kind of social interaction to support the development of their executive functions. But that is my only immediate reservation. I hope Braem, Hommel and others pursue the hypothesis that executive functions are cognitive gadgets, and, whatever the answer, that this line of enquiry has the benefits identified in their final paragraph. Stimulating research of this kind is exactly what I hoped CG would do.

\section{R5.2 Case studies}

\section{R5.2.1 Selective social learning}

Rathkopf \& Dennett encourage me - in a charmingly collegial way, but also with force - to reflect on the "benefits of embracing the messy middle", especially in relation to selective social learning. They argue that there are likely to be many varieties of social learning rule between those I describe as planetary and the explicitly metacognitive rules I describe as cook-like. There are likely to be many intermediate rules that involve increasing degrees of comprehension along with the competence. It is possible that Rathkopf \& Dennett overestimate the amount of comprehension I'm packing into cook-like social learning rules. Just as a cook does not need to know the chemistry that makes it wise to bake a cake at $180 \%$, a user of copy digital natives does not need to know the epistemology that makes it wise to learn IT skills from people born after 1985 . But Rathkopf \& Dennett's main point is well-taken: evolution is typically gradual, and therefore we should be on the look out for intermediate forms.

The question is: where should we look? It is easy to take any distinction between types of cognitive process and dream up a third (or fourth, or fifth...) type that shares characteristics with both. It is much harder to formulate new testable hypotheses; to conceptualise an intermediate type of cognitive process in a way that is both rooted in existing evidence and makes it possible to distinguish empirically between the new type and the types we already knew about. It is hard but, 
unless intermediates are conceptualised in this way, theorising about the evolution of mind will continue to float free of empirical science. I want research on the evolution of cognition to be messy in another sense - to get down and dirty with the data. With this kind of engagement as a cherished goal, I would look for intermediates between planetary and cook-like social learning rules in the cognitive science of implicit metacognition (Shea et al. 2014), not, like Rathkopf \& Dennett, in research on 'rational imitation'. Experiments by Beisert et al. (2012) suggest that, in both human infants and chimpanzees (Buttelemann et al. 2007), rational imitation effects are due to distraction. For example, a head movement is less likely to be copied when the model's hands are wrapped than when they're free, not because the subject understands wrapped hands to indicate lack of free choice, but because distraction by the wrapping procedure makes it less likely that the subject will attend to the head movement. If this is correct, if rational imitation effects are due to distraction, they are produced by wholesomely planetary social learning biases (Heyes 2016a). .

Like many others (e.g. Tennie), but in contrast with Whiten, I see the inheritance of behaviour via social learning in animals as importantly different from human culture because it is not cumulative; it does not afford cultural selection. However, in previous work my colleagues and I have given a straightforward answer to Whiten's question about how to test for explicit metacognition in non-linguistic creatures: "If, contrary to our hypothesis, non-human animals have system 2 metacognition, they should be able to learn that reward-seeking behaviour is successful after making decisions that are unlikely to be correct (low confidence) and unsuccessful after making decisions that are likely to be correct (high confidence). This could be tested by, for example, using a reverse transfer test after training in a wagering task" (Shea et al. 2014).

\section{R5.2.2 Imitation}

Del Giudice is right to point out that twin studies have limited value in parsing the contributions of nature, nurture and culture to cognitive development (e.g. Feldman \& Ramachandran 2018), and that, away from my home turf of experimental psychology and cognitive neuroscience, I misreported 
the results of a twin study of imitation. It was Hughes et al (2005), not McEwen et al (2007), who found the same correlation between identical and fraternal twins. McEwen et al found a .3 difference between the within-pair correlations, and concluded: "individual differences in imitation at age 2 years could be attributed to modest heritability, but mainly environmental influences" ( $p$. 485). Echoing a crucial point made by Braem \& Hommel about endophenotypes, McEwen et al. also noted: "The fact that $30 \%$ of the variance can be attributed to genetic factors could mean that genes directly influence individual differences in imitation mechanisms, although it is entirely possible that the impact is on more basic perceptual, attentional or motivational factors" (p. 485). Fortunately, the case for imitation as a cognitive gadget rests not on twin studies - which were not even mentioned in the chapter of $C G$ devoted to imitation - but on experimental data confirming predictions of the associative sequence learning (ASL) model, and indicating wealth of the stimulus.

In her deep and well-informed commentary, Powell argues that, even if the ASL model is right about the development of imitation, the resulting cognitive mechanism may be, not a cognitive gadget but a "cultural starting point"; not a mechanism favoured by cultural selection because it promotes cultural inheritance, but a mechanism, made possible by social elements of the genetic starter kit and dependent on social learning for its development, that acts as a platform for the evolution of true cognitive gadgets. I find this proposal very interesting indeed, and not only because it converges with work that Jonathan Birch (2017) and I are doing on 'the cultural evolution of cultural evolution'. Powell is acutely aware of the challenges inherent in explaining not only how cognitive gadgets get off the ground, in evolutionary and developmental time, but the subtle interplay between social practices and cognitive mechanisms as targets of cultural selection (see also McNamara \& Neha, Smaldino \& Spivey). I am not entirely convinced by Powell's evidence that parents' imitation of infants, and social partners' positive responses to being imitated, are sustained only by "incremental increases in the human genetic predisposition for social motivation and attention". For example, many of the studies she cites, which claim to show that infants and adults respond positively to being imitated, did not include adequate controls for contingency, and there is 
evidence that, when imitation and contingency are dissociated, it is the latter that makes us feel warm towards others (Catmur \& Heyes, 2013). But these reservations aside, Powell's subtle analysis has given me much to think about. I am grateful to her.

Tennie's planet-of-the-apes reflections on imitation were also enlightening. I love the idea that "ape imitation is a gadget lent to apes by humans", and I am intrigued by his evidence that imitation evolved only about 500 thousand years ago.

Whiten and I have a long, and usually friendly, history of disagreement about imitation. Instead of repeating answers to some of his "twenty questions" that I have offered in the past (e.g. Heyes 2016b), I would like to highlight a point of solid agreement between us: imitation of the topography of body movements (what Whiten calls "high fidelity copying") is important primarily for the inheritance of social, rather than instrumental, behaviour (Heyes 2013). Also, I am glad he drew attention to a key feature of the ASL model: it implies that imitation is compositional. Through social interaction (being imitated, synchronous action, mirror experience etc), the child builds up a repertoire, or vocabulary, of action units that can subsequently be imitated when they are encountered in novel sequences and configurations. Just as language users can understand sentences they have never heard before, imitators can copy compound body movements they have never seen before. Finally, Whiten is surely right that it would be valuable to have more information about the sources of imitogenic experience available to children in their everyday lives. However, evidence that children learn to imitate, in the manner proposed by the ASL model, is accumulating fast (e.g. de Klerk, Lamy-Yang \& Southgate, 2018).

\section{R5.2.3 Mindreading}

I particularly enjoyed the commentaries that focussed on mindreading (Apperly, Dominey, McNamara \& Nehu). While open to the idea that mindreading is culturally inherited, they identified patches where my treatment of the subject is "thin" (McNamara \& Nehu) and added valuable thickness. 
My reading of the evidence to date suggests that much of what is culturally inherited, at least in WEIRD societies, amounts to mental state concepts. However, I would not contest Apperly's proposal that, "in a long social apprenticeship", learning from others to identify relevant information is yet more important in the development of mindreading. Similarly, although I live on the information side of the information-understanding divide (see R1 above), and do not embrace the extended mind for day-to-day scientific use (see R2), I find great value in the ideas that mindreading is culturally inherited via narrative practice and analogical mapping (Dominey, Fenici \& Garofoli, Hutto 2007). Furthermore, I was educated by McNamara and Nehu's evidence of how "teaching and learning environments vary across cultures to provide children with context-specific opportunities to develop the cognitive abilities needed to thrive as adults". Their reference to "culture itself" implies that the domain of culture is exhausted by what I call grist - behaviour, beliefs, artefacts etc - whereas a primary aim of $C G$ is to show that distinctively human cognitive mechanisms are also cultural. However, that quibble did not dampen my enthusiasm as McNamara and Nehu directed us to rich seams of data from cultural psychology.

\section{R5.2.4 Language}

I need to think further about the many subtle and interesting points made by Dominey, but I am sympathetic to his view that language is a very special cognitive gadget. I do not believe that language is necessary for all gadget construction - for example, the ASL model implies that imitation can get going without it - and I take seriously the idea that language itself is rooted in associative learning. However, once language is in place, even with a toe hold, it enables the evolution and development of a wide array of other gadgets. If Dominey and I differ at all in the importance we assign to language, it is probably because he is preoccupied by sophisticated cultural grist - creation myths, mathematical concepts, the causal roles of mental states - whereas I am at least equally interested in the cultural inheritance of nonverbal social behaviour and motor skills. Verbal instruction is of more limited value in learning shibboleths - facial, postural and vocal gestures that 
distinguish one social group from another - and the skills involved in making and using tools (Stout \& Hecht, 2017).

Jablonka, Ginsburg \& Dor remind us that many peripheral mechanisms have been genetically specialised for language - "the innervation and musculature around the mouth, the larynx and the vocal cords, the unique function of the expanding muscles around the lungs" - and go on to say that "There is no reason to believe that the cognitive system, responsible for the activation and control of this physiology, somehow managed to remain unbiased towards it". Quite right, there is no reason to doubt that the mature cognitive system is biased for language. But the evidence surveyed in Chapter 8 of $C G$ provides many reasons to doubt that the biasing was done by selection operating on genetic variants. Research in cognitive science on the roles of domaingeneral sequence learning and social shaping in the development of language makes it fully plausible that, while genetic selection has done the lion's share on peripheral mechanisms, cultural selection has shaped the cognitive mechanisms responsible for language processing. If theorising about the evolution of human cognition is to be evidence-based, any claim that our minds are genetically specialised for language must, I believe, engage with that research.

\section{R5.2.5 Autobiographical memory}

Autobiographical memory was not one of my case studies but in their fascinating commentaries McNamara \& Neha and van Bergen \& Sutton showed that it deserves a central place in "an expanded cognitive science of gadgets". The combination of cross-cultural and intervention studies, clinical relevance, and hypotheses linking different gadget-generating social practices with ecological conditions, makes autobiographical memory rich territory for cultural evolutionary psychology. I hope future work will examine further how elaborative and repetitive reminising change not only what is remembered and when, but the computational processes of remembering. Autobiographical memory also presents an excellent opportunity to develop the idea of a compound gadget (van Bergen \& Sutton, Dominey). All gadgets are compounds in that, like any complex cognitive 
mechanism, they incorporate many subroutines. But are some gadgets compounds in a deeper sense - combinations of other gadgets, such as episodic memory and mindreading, that can function alone or, in different contexts, as a single system? Like all questions about the individuation or 'unitisation' of cognitive mechanisms, the answer is far from obvious and cannot be solved by intuition. The beauty of computational cognitive science is that it uses, not intuition or folk psychology, but empirical methods to find out about the structure and functions of the mind (Shallice \& Cooper, 2011). That is why, in $C G$, I recommend cognitive science as a valuable resource to anyone interested in human evolution.

\section{R6 Concluding remark}

While a part of me would have preferred 30,000 words "of closely reasoned adulation", what the commentators provide is much more invigorating and instructive. I am grateful to them all for reading the book, and offering critiques that will help cultural evolutionary psychology to identify more and better cognitive gadgets.

Acknowledgements

I am grateful to Max Coltheart, Martin Eimer, Noel Malcolm and Nick Shea. 
References not in the precis and commentaries

Beisert, M., Zmyj, N., Liepelt, R., Jung, F., Prinz, W., \& Daum, M. M. (2012). Rethinking 'rational imitation'in 14-month-old infants: A perceptual distraction approach. PLoS One, 7, e32563.

Birch, J. (2017). The Philosophy of Social Evolution. Oxford: Oxford University Press.

Block, N. (1995). The mind as the software of the brain. In E. E. Smith \& D. N. Osherson (Eds.), Thinking (pp. 377-425). Cambridge, MA: MIT Press.

Buskell, A. (2018). Causes of cultural disparity: Switches, tuners, and the cognitive science of religion. Philosophical Psychology, 31, 1239-1264.

Catmur, C. \& Heyes, C. M. (2013) Is it what you do, or when you do it? The roles of contingency and similarity in pro-social effects of imitation. Cognitive Science, 37, 1541-1552

Coltheart, M. (2002) Cognitive neuropsychology. In J. Wixted (Ed.) Stevens' Handbook of Experimental Psychology. Wiley. Pp 139-174.

Coltheart, M. (2012). The cognitive level of explanation. Australian Journal of Psychology, 64, 11-18. de Klerk, C. C., Lamy-Yang, I., \& Southgate, V. (2018). The role of sensorimotor experience in the development of mimicry in infancy. Developmental Science, e12771.

Del Giudice, M., Manera, V. \& Keysers, C. (2009) Programmed to learn? The ontogeny of mirror neurons. Developmental Science, 12, 350-63.

Feldman, M. W., Odling-Smee, J., \& Laland, K. N. (2017). Why Gupta et al.'s critique of niche construction theory is off target. Journal of Genetics, 96, 505-508.

Feldman, M. W., \& Ramachandran, S. (2018). Missing compared to what? Revisiting heritability, genes and culture. Philosophical Transactions of the Royal Society B: Biological Sciences, 373(1743), 20170064.

Gupta, M., Prasad, N. G., Dey, S., Joshi, A., \& Vidya, T. N. C. (2017). Niche construction in evolutionary theory: the construction of an academic niche?. Journal of Genetics, 96, 491-504. Heyes, C. M. (2016a). Born pupils? Natural pedagogy and cultural pedagogy. Perspectives on Psychological Science, 11, 280-295. 
Heyes, C. M. (2016b). Homo imitans? Seven reasons why imitation couldn't possibly be associative. Philosophical Transactions of the Royal Society: B., 371: 20150069

Shallice, T., \& Cooper, R. (2011). The Organisation of Mind. Oxford: Oxford University Press.

Shea, N., Boldt, A., Bang, D., Yeung, N., Heyes, C. M. \& Frith, C. D. (2014). Supra-personal cognitive control and metacognition. Trends in Cognitive Sciences, 18, 186-193.

Stout, D., \& Hecht, E. E. (2017). Evolutionary neuroscience of cumulative culture. Proceedings of the National Academy of Sciences, 114, 7861-7868. 